\title{
MOVILIDAD RESIDENCIAL EN EDADES AVANZADAS EN ESPAÑA: UNA PERSPECTIVA METROPOLITANA
}

\author{
Isabel Pujadas Rúbies ${ }^{1}$ \\ JORDI BAYONA-I-CARRASCO ${ }^{2}$ \\ Miguel Rubiales PéreZ ${ }^{3}$
}

\begin{abstract}
RESUMEN - En las áreas metropolitanas de Barcelona y Madrid, las dos mayores regiones metropolitanas de España, las migraciones internas de carácter residencial protagonizadas por mayores de 80 años doblan sus intensidades en apenas dos décadas. Este crecimiento se produce de forma similar en el conjunto de España, y se relaciona con la pérdida de independencia domiciliar producto de la edad. Desde un punto de vista territorial, estas migraciones se caracterizan por una sobrerepresentación de las salidas desde las ciudades centrales de las dos metrópolis, con una especialización de algunos de los municipios periféricos en su recepción, principalmente de aquellos donde la tipología de la vivienda predominante es la unifamiliar. Las migraciones previas de los hijos en un entorno metropolitano, además de la fijación de instituciones para mayores en dichos municipios, explican unas pautas que, por otro lado, son muy parecidas en Barcelona y Madrid.
\end{abstract}

Palabras clave: Migración interna; población en edades avanzadas; características sociodemográficas; pautas territoriales.

ABSTRACT - Residential mobility of old people in Spain: A metropolitan PERSPECTIVE. This paper analyses the internal migration of the elderly in the Barcelona and Madrid metropolitan areas. The population with more than 80 years experienced an increase in the intensity of their mobility over the past two decades. This growth is distributed throughout the country and associated with an age related autonomy loss. From a territorial point of view, the main traits of these migrations are: an overrepresentation of exits from the central cities of both metropolises and the concentration of arrivals in some peripheral

Recebido: Outubro 2014. Aceite: Janeiro 2016.

1 Catedrática de Geografía, Departament de Geografia, Universitat de Barcelona. E-mail: ipujadas@ub.edu

2 Profesor Asociado, Departament de Geografia, Universitat de Barcelona, e investigador del Centre d'Estudis Demogràfics. E-mail: jordibayona@ub.edu

3 Becario FPU del Ministerio de Educación, Departament de Geografia, Universitat de Barcelona. E-mail: mrubiale@gmail.com 
municipalities, specifically in those where single-family dwellings are the predominant housing typology. These features, in both Barcelona and Madrid, can be explained by the prior migration of children and the regional location of nursing homes.

Keywords: Internal migration; sociodemographic characteristics; territorial patterns; old ages.

RESUMO - MOBILIDAde RESIDENCIAL EM IDADES MAIS AVANÇADAS EM ESPANHA: uma Perspectiva metropolitana. Nas áreas metropolitanas de Barcelona e Madrid, as duas maiores regiões metropolitanas de Espanha, as migrações internas de tipo residencial feitas pelos maiores de 80 anos dobram as suas intensidades em apenas duas décadas. Esse crescimento é feito de modo semelhante ao conjunto da Espanha, e relaciona-se com a perda de independência domiciliar, produto da idade. Desde uma ótica territorial, essas migrações caracterizam-se por uma sobrerepresentação das saídas das cidades centrais das duas metrópoles, e com a especialização de alguns municípios da periferia na sua receção, principalmente de aqueles onde a tipologia de moradia predominante é a unifamiliar. As migrações prévias dos filhos num círculo metropolitano, além do estabelecimento de instituições para idosos naqueles municípios, explicam que as orientações que fazem tornem semelhantes os casos de Barcelona e Madrid.

Palavras-chave: Migrações interiores; população idosa; características sócio-demográficas; pautas territoriais.

RÉSUMÉ - LA Mobilité RÉSIDENTIELle dES PERSONNES ÂGÉES EN ESPAgNE: UNE perspective métropolitaine. Dans le contexte de Barcelone et de Madrid, les deux plus grandes régions métropolitaines d'Espagne, la migration interne résidentielle des personnes de plus de 80 ans a doublé d'intensité en deux décennies. Cette croissance est liée à la perte de l'autonomie des personnes âgées et se produit de façon similaire dans toute l'Espagne. Du point de vue territorial, ces migrations se caractérisent par la sortie des capitales métropolitaines et la concentration des arrivées dans quelques municipalités périphériques, où le type prédominant des logements est la maison familiale. Ces caractéristiques, très semblables à Barcelone et Madrid, pourraient être expliquées par la migration antérieure des enfants vers les régions métropolitaines et par l'installation des maisons de retraite dans les municipalités périphériques.

Mots clés : Migration interne; caractéristiques sociodémographiques; modèles territoriaux; âge avancé.

\section{INTRODUCCIÓN, OBJETIVOS E HIPÓTESIS DE TRABAJO}

En un reciente proyecto de investigación, ya finalizado, que abordaba de forma comparativa las dinámicas demográficas urbanas en las regiones metropolitanas españolas de Barcelona y Madrid, y de forma más particular la evolución de las pautas de movilidad residenciali, uno de los resultados más significativos y que se producía con similar magnitud en ambas áreas de estudio era el aumento, de forma importante y en apenas un par de décadas, de la intensidad de la movilidad residencial producida en las edades más avanzadas (fig. 1). Estos resultados indicaban como en ambas regiones las tasas de migración interna se llegaban incluso a doblar entre la población que superaba los ochenta años de edad 
(Bayona \& Pujadas, 2014; Pujadas \& Bayona, 2015). Por si fuera poco, el incremento de estas tasas era incluso mayor que el observado en las edades más móviles, diferenciándose cada vez más de la movilidad observada entre los 50 y los ochenta años de edad. De esta forma, se llega a producir una edad modal secundaria de la movilidad residencial alrededor de los noventa años, con valores de intensidad que superan tasas del 20 por mil anual. La coincidencia de los perfiles por sexo y edad de esta movilidad, en cuanto se observa una mayor intensidad entre las mujeres, es otra de las características compartidas entre Barcelona y Madrid.
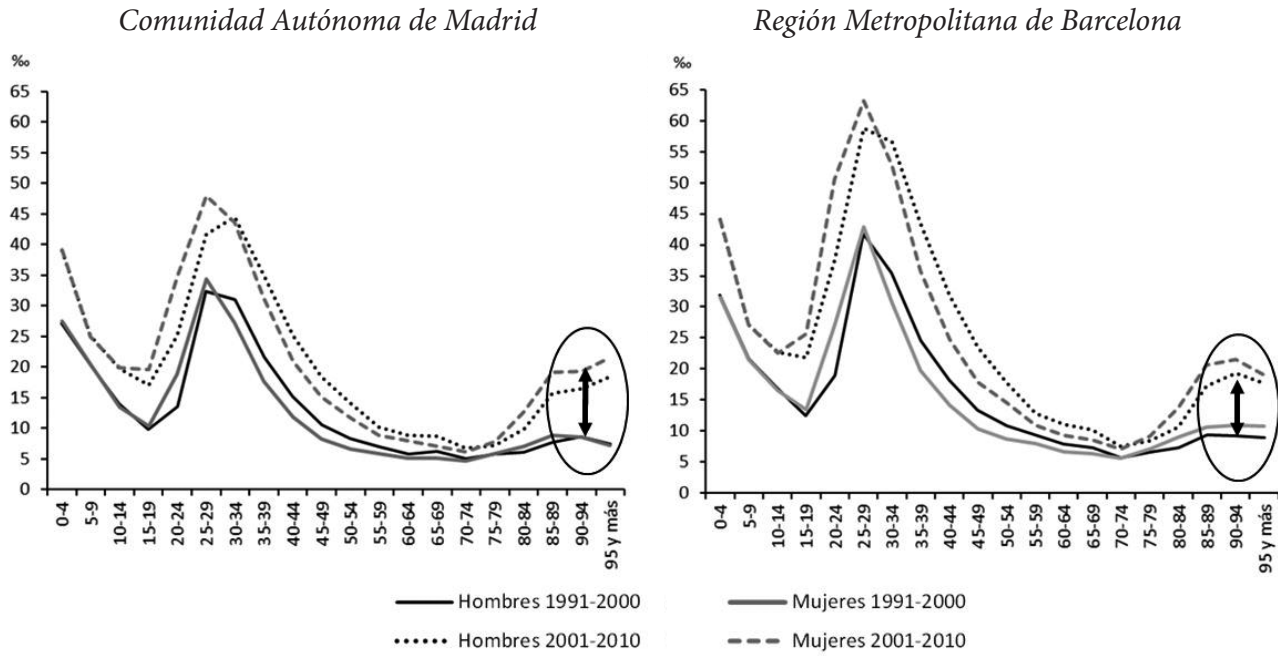

Fig. 1 - Tasas de movilidad interna por sexo y edad, Comunidad Autónoma de Madrid y

Región Metropolitana de Barcelona, 1991-2000 y 2001-2010.

Fig. 1 - Internal mobility rates by sex and age, Autonomous Community of Madrid and Barcelona

MetropolitanRegion 1991-2000 and 2001-2010.

Fuente: Elaboración propia, con datos de la Estadística de Variaciones Residenciales (EVR), 1991-2010, del Censo de población (1991) wwyel Padrón Continuo de población, del Instituto Nacional de Estadística (INE).

Estos incrementos observados más la coincidencia del mismo proceso en las dos áreas urbanas justifica el presente trabajo, que trata con mayor profundidad esta situación identificada. Inicialmente, nos planteamos varias hipótesis que pueden servir para interpretar dichas variaciones en la intensidad, en unas edades donde el cambio de municipio se relaciona con toda seguridad con la pérdida de independencia domiciliar producto del envejecimiento y de la propia edad. En primer lugar, cabe considerar que la intensificación de dicha movilidad puede estar indicando una migración residencial relacionada con un cambio de residencia previo de los hijos (debido al proceso de suburbanización, ya que abordamos el estudio de dos regiones metropolitanas donde este proceso se desarrolla desde inicios de los años ochenta), y por ende, de un proceso de reagrupación familiar relacionado con la edad y que implica un cambio domiciliario y municipal. También, de forma paralela y no excluyente, el crecimiento de la movilidad residencial podría indicar la posible mayor institucionalización de este colectivo en la actualidad en relación a décadas precedentes, y de la existencia de residencias para la tercera edad en municipios distintos al de residencia actual (en zonas urbanas más dispersas, por ejemplo), con lo que el internamiento en una de estas instituciones debería comportar un desplazamiento residencial. Sin descartar otras hipótesis, creemos que ambas posibilidades deben copar la gran mayoría de cambios residenciales registrados en estas edades. 
En función de los perfiles etarios obtenidos en la figura 1, este trabajo centra la atención en los mayores de 80 años de edad. De ellos, se analizan sus migraciones internas en el contexto de las dos mayores regiones metropolitanas de España, la de Madrid (a partir de ahora CAM, ya que se utiliza el conjunto de la Comunidad Autónoma de Madrid) y la de Barcelona (Región Metropolitana de Barcelona, RMB). Los tres principales objetivos de esta investigación se resumen en conocer los siguientes aspectos:

1) Si el aumento de las tasas migratorias intra-metropolitanas en las edades más avanzadas es un fenómeno básicamente metropolitano o bien se produce por igual en todo el país;

2) Si, en comparación al resto de edades, se da la existencia en el contexto metropolitano de pautas territoriales específicas; y finalmente,

3) La existencia de singularidades socio-demográficas diferenciales de aquellos mayores que se desplazan a escala metropolitana.

\section{FUENTES ESTADÍSTICAS Y ASPECTOS METODOLÓGICOS}

Este trabajo realiza una aproximación al fenómeno de la movilidad interna desde una perspectiva cuantitativa. Para ello se utilizan dos fuentes estadísticas de características muy diferentes. En primer lugar, se analizan los datos de la Estadística de Variaciones Residenciales (EVR), fuente derivada del Padrón Continuo de población ${ }^{\text {ii }}$, con los que se estudia la intensidad de los cambios residenciales internos y su evolución, y al mismo tiempo, las pautas territoriales presentadas por la movilidad a escala provincial y metropolitana. Para ello se han delimitado los grupos de edad pertenecientes a los mayores de 80 años, y se emplean datos de una serie estadística de 27 años que se remontan a 1988, primer año con microdatos disponibles, hasta el reciente 2014. El umbral de edad utilizado se define a partir de la observación empírica de las tasas de movilidad representadas en la figura 1, ya que si bien estas aumentan en todas las edades por encima de la jubilación, es en estos grupos de edades más avanzadas donde el crecimiento de las tasas es mayor, incluso dibujando un nuevo perfil de la movilidad residencial en relación al crecimiento experimentado en estas edades. En este sentido, cabe recordar que no existe un consenso preestablecido sobre la edad cuando se estudia la movilidad residencial de los mayores o de los hogares de los mayores. En la creciente bibliografía sobre el tema, encontramos como en la mayoría de estudios se delimita la edad muy tempranamente, en algunos casos incluso a partir de los 50 años de edad (Tatsiramos, 2006; Angelini \& Laferrère, 2012); de los 55 años (Puga, 2000); o más usualmente utilizando la edad de jubilación (Fernández-Carro \& Evandrou, 2013). En nuestro caso, la atención se centra en un subgrupo muy específico de la población, que no acostumbra, por sí sólo, a establecerse como objetivo de estudio.

Desde un punto de vista territorial, el marco de análisis es el entorno metropolitano $o^{\mathrm{iii}}$ (fig. 2), entendiendo que los procesos que puedan producirse a esta escala difieren de los observados en otros ámbitos geográficos, como puede ser el caso del mundo rural. De esta manera se han analizado las dos mayores regiones metropolitanas de España, las construidas en torno de las ciudades de Madrid y de Barcelona. Para el caso de Barcelona se emplea la denominada Región Metropolitana de Barcelona (RMB), que cuenta con 164 municipios en $3.236 \mathrm{~km}^{2}$ y con 5.028.258 habitantes a inicios de 2015, área que fue delimitada durante los años sesenta y que históricamente, desde entonces, se ha utilizado como unidad de análisis metropolitana preferente. En el caso de Madrid, en cambio, y dada la inexistencia de una delimitación consensuadamente utilizada, empleamos el conjunto de la Comunidad Autónoma de Madrid (CAM), con 179 municipios en $8.022 \mathrm{~km}^{2}$ y 6.436.996 habitantes en 2015. Ambas unidades se emplean habitualmente como definición de las respectivas regiones metropolitanas (Nel·lo, 2004; Méndez, $2008)^{\text {iv }}$, aunque en sus periferias la dinámica metropolitana no siempre esté presente (Sierra Norte en Madrid, comarca del AltPenedès en la RMB). Conjuntamente suman 11,5 millones de residentes, lo que representa casi una cuarta parte de la población de España. 
En segundo lugar se emplea el último Censo de población y viviendas de 2011. A partir del análisis de los microdatos se estudia con mayor detalle las características sociodemográficas de aquellos mayores de edad que han realizado, durante los últimos años, un movimiento migratorio interno. En este análisis, y para la provincia de Barcelona, se utiliza el total provincial en lugar de la RMB. Esto es debido a la no disponibilidad de una desagregación municipal suficiente para reconstruir unidades infraprovinciales $^{v}$. En cambio, se mantiene la unidad de análisis para la CAM, al coincidir con la provincia. Con los microdatos del Censo de 2011 se ha aplicado un análisis de agrupación en árboles, metodología estadística que permite identificar la estructura subyacente de los datos. Se han utilizado seis variables interpretativas: el sexo, la estructura del hogar, el nivel de estudios, el tamaño del municipio, la nacionalidad y el tamaño de la vivienda. Cada subdivisión de los datos se realiza intentando que los grupos resultantes sean lo más homogéneos posibles internamente.

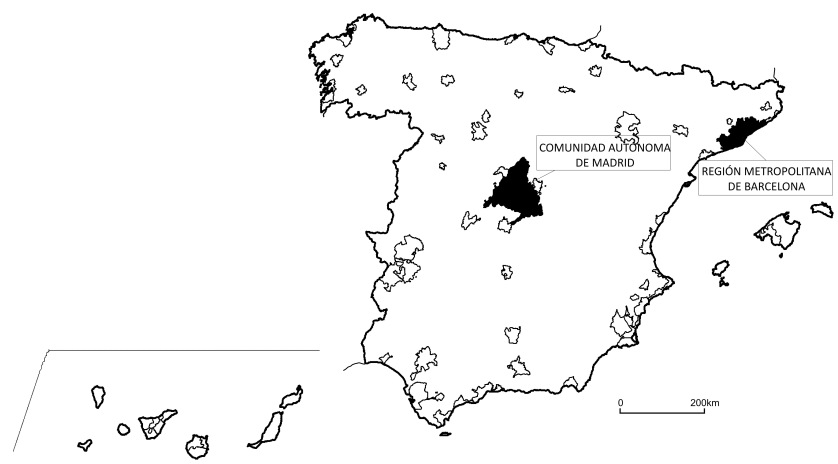

Fig. 2 -Localización de las principales regiones metropolitanas españolas.

Fig. 2 - Location of the main Spanish metropolitan regions.

Fuente: Elaboración propia a partir del Atlas Estadístico de las Áreas urbanas en España, 2006

En la comparación entre ambas fuentes debemos tener en cuenta como el Censo y las EVR registran de forma diferente las migraciones, y en especial las migraciones internas. Por ejemplo, para el conjunto de España el Censo de población de 2011, con fecha de referencia a 1 de noviembre, registra 690 mil migrantes internos durante el último año. Por su parte, la Estadística de Variaciones Residenciales del año 2011 (es decir, con datos referidos a todo el año 2011) cuenta hasta 1.650 .000 migraciones internas (un 26,6\% de las cuales de extranjeros), 15.030 y 45.535 migraciones según las fuentes respectivas si se considera la movilidad de los mayores de 80 años en España. Como se puede observar, los datos obtenidos por una fuente estadística (EVR) triplican la intensidad de la otra (Censo) entre la población estudiada. Parte de las diferencias se deben a que en el Censo solamente se registran las migraciones internas de los residentes en viviendas familiares, ya que el Censo no da información de la movilidad hacia establecimientos colectivos (y que podría explicar parte de la menor movilidad en las edades aquí estudiadas). Estas diferencias fueron ya apreciadas con el Censo de 2001, y fueron atribuidas básicamente a una falta de respuesta y a la mala imputación por parte del INE de estos casos, pero también, en menor porcentaje, a migraciones repetidas y falsas migraciones captadas por las EVR (Ródenes \& Martí, 2009), aunque estas últimas representan un porcentaje poco importante y no justifican por sí solas las diferencias.

Una de las principales problemáticas existentes, si consideramos la fiabilidad de los datos empleados, es la existencia o no de un empadronamiento al realizar el cambio de municipio, es decir, hasta qué punto se produce la comunicación estadística del desplazamiento. Esta situación, que infravaloraría la 
movilidad total, podría afectar más a los mayores de edad, en cuanto el cambio de domicilio legal podría incluso tener repercusiones sobre los impuestos a pagar por el domicilio habitual en caso de fallecimiento y en el proceso de herencia. Algunos de los resultados del Censo de 2011, referidos a los residentes en establecimientos colectivos $^{\text {vi }}$ añaden mayor confusión a esta situación: sólo el 61,6\% de los que habitan en residencias para mayores se encontraban realmente empadronados en éstas. Además, parecen existir ciertas particularidades geográficas, ya que este porcentaje es más importante en la provincia de Barcelona (el 72,1\%, 25.137 de 34.849 personas) que en Madrid (el 61,5\%, 23.303 de 37.887 personas). Esto incluso indica un subregistro de las Estadísticas de Variaciones Residenciales (EVR) de esta movilidad, con lo cual las diferencias al compararlas cifras de movilidad con el Censo serían aún mayores. Desconocemos, en cambio, el posible subregistro si esta movilidad se realiza en dirección de hogares de familiares.

\section{MARCO TEÓRICO: EL CRECIENTEINTERÉS POR LA MOVILIDAD EN EDADES AVANZADAS}

Rogers (1988), en su modelo de movilidad, identificaba la existencia de una pauta de intensidad de las migraciones según la edad delos individuos, donde las edades relacionadas con el proceso de jubilación (alrededor de los 65 años) experimentaban un ligero repunte de la intensidad, para un último incremento de la movilidad relacionada con la pérdida de independencia (producto de la enfermedad) o por la defunción de uno de los cónyuges, que se produciría en edades muy avanzadas. Estos perfiles de la movilidad se caracterizan por su estabilidad, observándose pequeñas variaciones entre países, que generalmente se producirían entre las edades jóvenes y adultas-jóvenes (Bernard et al., 2014).

Estas migraciones en edades avanzadas no acostumbran a ser numéricamente muy destacadas, ya que se observan, en estas edades, otros tipos de movilidad. Lardíes (2010), por ejemplo, y a partir de un estudio cualitativo para el conjunto de los desplazamientos de la población mayor, indica como la existencia de familiares y especialmente de los nietos ejerce una influencia muy significativa en los desplazamientos residenciales, encontrando una preferencia por los movimientos pendulares en relación a los desplazamientos definitivos. La multiresidencia, a la que se refiere esta opción, no queda bien recogida en los censos ni en los padrones de población, opción de movilidad a la que no podremos aproximarnos.

Por su parte, Fernández-Carro y Evandrou (2013) apuntan como la mayor parte de los mayores de 65 años permanecerán residiendo de forma estable en sus propios domicilios hasta que el traslado a una institución u hogar dependiente es inevitable por deficiencias severas en el estado de salud. Esta preferencia por envejecer en el propio domicilio es lo que se conoce como "Ageing in place", y es el modelo preferido de envejecimiento en el contexto europeo (Rojo et al., 2001, Costa-Font et al., 2009).

Dentro de este modelo, las características sociodemográficas de los individuos y de la vivienda donde residen aparecen como factores determinantes para conocer su posible movilidad. La propiedad mayoritaria de la vivienda de algunas de estas generaciones, más las pensiones que cobran del Estado (lo que les garantiza un mínimo de estabilidad), producen que mantengan su residencia habitual y adapten ésta a las condiciones físicas cambiantes, posponiendo la movilidad (Bonvalet \& Ogg, 2008). El tránsito entre propiedad y alquiler en las edades más avanzadas, en cambio, se asocia a aquellos que viven solos, especialmente cuando se relaciona con la viudedad o separación (Herberset et al., 2014).

El análisis longitudinal o de curso de vida aporta también una perspectiva interesante de análisis, en cuanto los cambios residenciales que se producen en la vejez deben relacionarse con el historial migratorio y los cambios de residencia previos del individuo (Puga, 2004), sin los cuales es difícil de entender las migraciones que se producen en la actualidad (Gobillon \& Laferrére, 2006).

Existe, también, un consenso sobre la creciente importancia de los desplazamientos entre los mayores. Puga (2000), considerando en este caso a los mayores de 55 años, estima que uno de cada cinco de ellos realizará, en un futuro próximo, un movimiento migratorio en edades avanzadas (Puga, 2004a y 
2004b). En estos trabajos se observa ya la existencia, a partir de los 75 años de edad, de un aumento de las probabilidades de migrar, edades donde a veces la migración no es "una opción, sino una imposición creada por la soledad o la discapacidad” (Puga, 2000). Desde una perspectiva metropolitana García-Coll et al. (2016), observan también este crecimiento de las tasas de migración interna en edades muy avanzadas, geográficamente importante en los municipios con un hábitat más disperso de la región, aunque interpretan este crecimiento como un retorno al centro metropolitano relacionado con la pérdida de la independencia domiciliar y el mayor número de servicios de las ciudades más densas.

Abellán y Rojo (1997), y para la ciudad de Madrid, identifican una tipología de movimientos observados, pudiéndose estos agrupar en cuatro grandes flujos: retorno, movilidad a zona de amenidad, desplazamiento local e institucionalización. En todo caso, un porcentaje importante de los mayores cambia de residencia en Europa, con la cifra más reciente que se eleva al 20\% de los mayores de 65 años (Fernández-Carro, 2012).

Finalmente, cabe considerar además como este aumento de la movilidad, en el caso de España, se ha venido produciendo al mismo tiempo que crece la demanda de cuidadoras inmigrantes (Rodríguez et al., 2010, Domingo \& Houle, 2005), cuyo efecto es posiblemente el contrario, el de mantener a la persona de edad en su mismo entorno domiciliar, en contra del desplazamiento, facilitando lo que se conoce como "ageing in place", o el envejecimiento en un mismo domicilio.

\section{LAS MIGRACIONES INTERNAS EN EDADES AVANZADAS: EVOLUCIÓN TEMPORAL Y TERRITORIAL}

Desde que se dispone de datos anuales sobre los cambios de domicilio entre municipios, el crecimiento de las migraciones en el interior del país protagonizadas por mayores de 80 años es constante, multiplicándose por 8 el número de cambios municipales registrados. En el año 1988, en España, poco más de 5 mil personas eran empadronadas en un nuevo municipio a estas edades (5.574 casos, sólo el 0,95\% de los 589.087 migraciones internas contabilizadas en España). Desde entonces el crecimiento observado es constante y lineal, hasta alcanzar en 2014 un total de 45.733 migraciones y un peso del $2,93 \%$ de la movilidad global, aunque el número de migraciones es similar al de 2007, mientras aumenta ligeramente el peso sobre la movilidad total (fig. 3, derecha).

Parte del crecimiento observado en cifras absolutas se debe, obviamente, a un efecto estructura, por el continuado envejecimiento que se produce entre la población española. La evolución de los datos censales y padronales dan cuenta de este fenómeno: en 1991, los mayores de 80 años sumaban 1.147.490 personas. Años más tarde, y con cifras del padrón, a enero de 2015 se contabilizan 2.726 .076 personas que superan los 80 años de edad. En 25 años la cifra de mayores se multiplica por 2,4 y se añade 1,5 millones de personas, evidenciando el aumento de la longevidad en un país que ostenta una de las esperanzas de vida más elevadas a nivel mundial: 80,1 años para los hombres y 85,6 para las mujeres. En términos relativos la evolución es similar, si en 1991 eran el 2,95\% de la población española en 2015 representan el 5,85\%. Además, esta dinámica se ha producido en el período de mayor crecimiento de la población española, coincidiendo con el llamado boom migratorio internacional (Bayona, 2008; Domingo \& Cabré, 2015), con la llegada de seis millones de nuevos residentes, en gran parte jóvenes. De esta forma, España crece en los años considerados desde los 38.872.268 habitantes a los 46.624.382 (aunque antes de la crisis económica se llegaron a superar los 47 millones), con una tasa de crecimiento anual que se sitúa en valores del 0,77\%. Esta misma tasa, para los mayores de 80 años, se sitúa muy por encima, en el 3,71\%. A pesar del fuerte crecimiento de la población española producto del boom migratorio internacional, el proceso de envejecimiento ha continuado su curso con una elevada intensidad, eso sí, con un importante freno a su visibilidad debido a la emergencia de otros flujos migratorios, como es el caso de las migraciones internacionales. 
Volviendo a la movilidad residencial, es significativo como desde 1988 prácticamente se triplica la importancia de la movilidad en estas edades, a pesar de que estamos hablando de cifras modestas. Si consideramos un grupo etario más amplio, los mayores de 65 años, la movilidad experimentada, a pesar de conocer también un crecimiento, no muestra este continuo incremento (especialmente en el último lustro, como indican Parreño et al., 2014), como lo denota su participación sobre la movilidad total, con máximos a finales de los noventa y con una evolución más relacionada con la coyuntura económica del momento (fig. 3, izquierda). De hecho, la propia figura 1 muestra cómo, para las regiones metropolitanas de Barcelona y Madrid el crecimiento de la movilidad justo en las edades de la jubilación, alrededor de los 65 años, es la más baja de todas las edades.

65 años y más

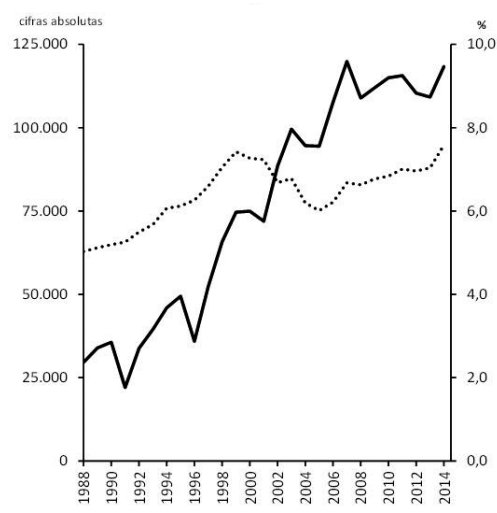

- Migraciones
80 años y más

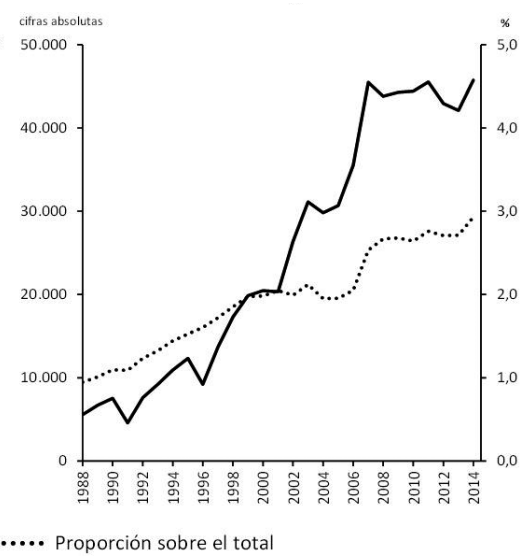

Fig. 3 -Evolución de las migraciones internas en España de los mayores de 65 y 80 años, 1988-2014.

Fig. 3 - Evolution of internal migrations in Spain of over 65 and 80 years, 1988-2014. Fuente: Estadística de Variaciones Residenciales, 1988-2014, con datos del Instituto Nacional de Estadística (INE)

2001-2002

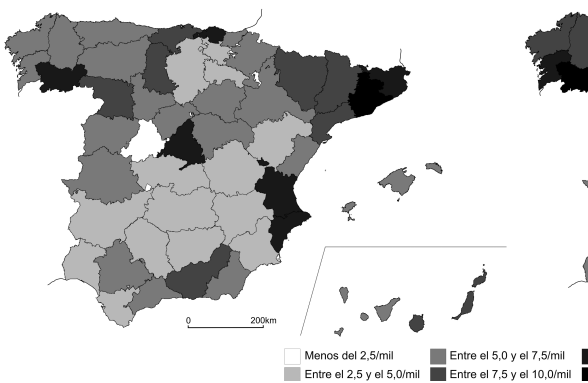

2013-2014

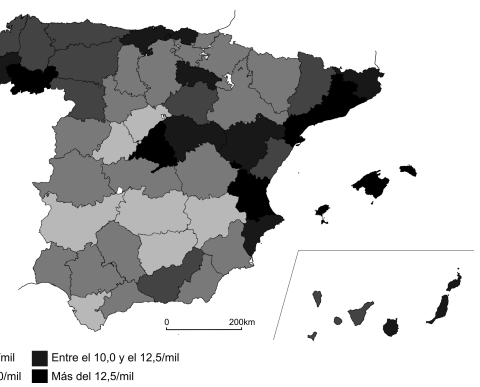

Fig. 4 - Tasas de movilidad intra-provincial en España de los mayores de 80 años.

Fig. 4 - Rates of intra-provincial mobility in Spain for over 80 years.

Fuente: EVR, y Padrón Continuo de población a 1 de enero de 2002 y 2012, con datos del INE 
Para establecer las pautas a nivel provincial, se ha comparado las tasas de movilidad intra-provinciales del grupo estudiado en dos momentos, en el bienio 2001-2002 (en pleno momento de expansión y antes de la etapa de mayor crecimiento del boom migratorio) y en el 2013-2014 (fig. 4), últimos años disponibles. Los resultados indican un crecimiento general de la movilidad interna intra-provincial de los mayores, situación que se observa en 42 de las 50 provincias analizadas. En las ocho restantes, el descenso siempre es poco importante, a excepción de Huesca, y en menor medida de Segovia y Palencia, mientras que en la mayoría de provincias se observan crecimientos de fuerte intensidad. Con valores por encima de Barcelona y Madrid sólo se sitúa una provincia, Ourense, aunque si bien Madrid experimenta un crecimiento importante de la movilidad (del 10 al 14,8 por mil), en Barcelona apenas este crecimiento es relevante (14,1 a 15,8 por mil). Esta última situación contrasta con los resultados obtenidos a nivel metropolitano, donde el crecimiento observado en la figura 1 era significativo. Esto significa un cambio en las destinaciones de los migrantes, si en el primer bienio se superaría normalmente el límite metropolitano, en el segundo las migraciones se producirán mayoritariamente dentro de los límites de la RMB. Por otro lado, estos resultados indican como más allá del efecto estructura, existe un crecimiento de la intensidad de las migraciones internas en las edades estudiadas, que se produce en todo el país.

2001-2002

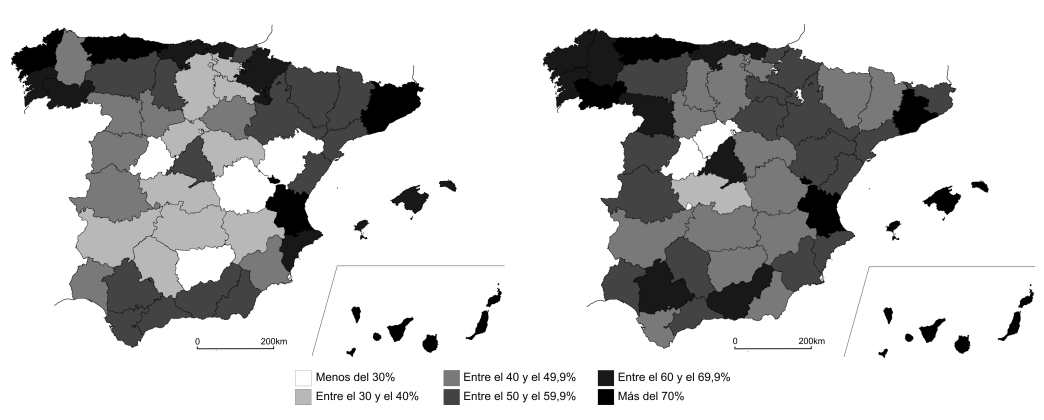

Fig. 5 - Proporción que representan las migraciones intraprovinciales sobre el total de migraciones internas en España, mayores de 80 años, 2001-2002 y 2013-2014.

Fig. 5 - Percentage of aged population (more than 80 years) over intra-provincial migrations, 2001-2002 and 2013-2014.

Fuente: Estadística de Variaciones Residenciales, 2001-2002 y 2013-2014, con datos del INE

En segundo lugar, y en relación con la movilidad total interna, se ha considerado en la figura 5 el peso de los movimientos intra-provinciales sobre el total de movimientos internos en el conjunto de España, para los mismos intervalos temporales. Los resultados nos indican qué provincias contienen la mayoría de migrantes internos, y cuales, en cambio, envían migrantes hacia otras zonas o provincias, así como la evolución en el tiempo. De esta forma, se observa como las provincias más pobladas y urbanas son las que más capacidad tienen de contener un mayor grado de movilidad (es el caso de Barcelona y Valencia), situación que se reproduce en menor medida en Madrid (donde la suburbanización llega a superar los límites provinciales y se extiende en dirección a Guadalajara o Toledo, por ejemplo). Las provincias insulares también experimentan cierto efecto de isolación, con una elevada autocontención migratoria en estas edades. Las provincias colindantes a Madrid son, en cambio, las que menor poder de retención muestran, producto en parte de la atracción migratoria ejercida por la capital. El norte de España y la costa mediterránea son también puntos de elevada contención de la movilidad. En cambio, usualmente se ha apuntado como en Madrid y en el País Vasco las migraciones de retorno ligadas a la 
edad de jubilación han sido siempre importantes (Recaño, 2004), situación que aquí no se observa debido a que esta migración, mayoritariamente, se produciría en edades por debajo de las aquí estudiadas. En cambio, se observa el efecto contrario, aquellas zonas de mayor emigración histórica retienen menos a sus mayores (es el caso de las dos castillas), mientras que el norte del país tiene un importante efecto de autocontención. Entre ambos periodos las pautas son similares, aunque el contexto general es del aumento de la autocontención.

\section{La movilidad a escala metropolitana}

Desde la perspectiva metropolitana (fig. 6) sólo se utilizan los movimientos migratorios producidos dentro del mismo ámbito, sin tener en cuenta movimientos realizados hacia otras zonas de España (o de la provincia, sea el caso de la RMB). A pesar de ello, en primer lugar cabe apuntar como la participación de los mayores en la movilidad interna es ligeramente superior a la del conjunto de España que mostrábamos en la figura 3 (donde se consideraba todas las migraciones internas), porcentaje que aumentaría si se considerara el resto del país. En el caso de la RMB, por ejemplo, el número de desplazamientos protagonizados por mayores de 80 años se multiplica por 8 desde el año 1988, de los escasos 455 desplazamientos residenciales registrados en 1988 a los 4.360 recogidos en 2014, con un ascenso desde el 0,9\% de la movilidad total al 3,2\% de ésta. Además, si la movilidad global en la RMB se encuentra estancada desde 2003 (Pujadas et al., 2016), en cambio la movilidad de los mayores sigue en constante aumento. Para la Comunidad Autónoma de Madrid los resultados van en la misma dirección, aunque el crecimiento de la movilidad del grupo estudiado es incluso más importante, alcanzando a representar valores superiores al 3,5\% de la movilidad total. En ambos casos, la proporción de migraciones aquí observadas es ligeramente superior a la media estatal, con una evolución similar y que a partir de 2011 se encuentra ligeramente por encima (fig. 7).

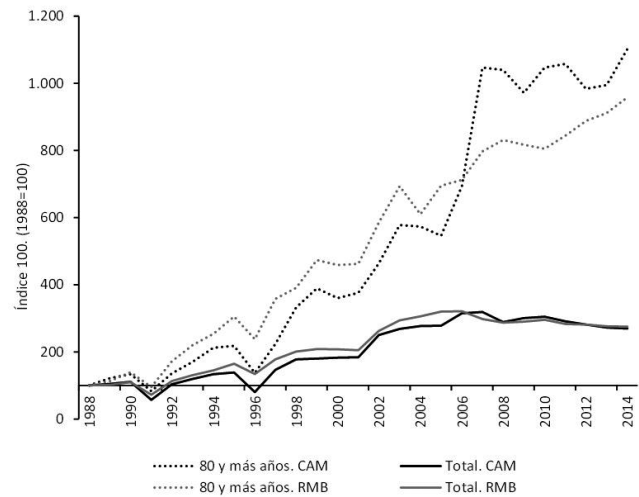

Fig. 6 - Evolución de las migraciones de los mayores de 80 años y del total. Índice 100 (1988=100), 1988-2014.

Fig. 6 - Evolution of migrations of general and over 80 years population.

Index $100(1988=100), 1988-2014$.

Fuente: EVR, 1988-2014, con datos del INE

Utilizando las tasas de migración, se constata como la movilidad a estas edades es inferior a la del conjunto de la movilidad (fig. 8), ya que los cambios migratorios internos siguen produciéndose entre la población joven, mayoritariamente. Lo que la figura 8 nos dibuja es un comportamiento diferencial entre Barcelona (donde las tasas se mantienen a pesar de la crisis) y Madrid, con un descenso importante durante 
los últimos años, desconociendo, con los datos disponibles, el motivo de esta evolución. En ambos casos, el año 2014 representa un ligero repunte de la movilidad entre los mayores, mientras el resto de la movilidad sigue sin presentar alteraciones. En ambos casos se muestran tasas similares, del 15,2 por mil en la CAM y del 16,2 por mil en la RMB (por valores de 22,0 y 27,1 por mil respectivamente entre el total).

Finalmente, indicar que se trata, además, de un crecimiento de la movilidad donde no han participado apenas los residentes extranjeros ${ }^{\text {vii }}$, a diferencia de lo que ha sucedido con la movilidad interna del resto de la población en España, que no se puede explicar sin considerar que ha sucedido con los inmigrantes extranjeros, y donde la dinámica ascendiente ininterrumpida contrasta con la estabilización de la movilidad residencial de los españoles desde finales del primer quinquenio del siglo.

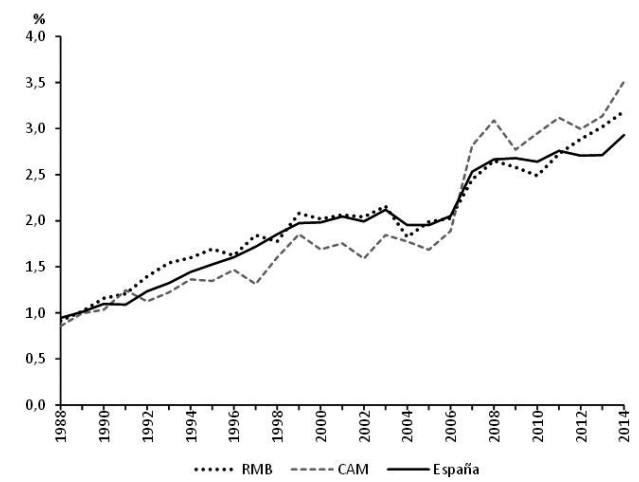

Fig. 7 - Peso de los cambios migratorios realizados por mayores de 80 años sobre el total de migraciones internas, RMB, CAM y España, 1988-2014.

Fig. 7 - Weight of migratory changes made by population over 80 years over total internal migration, RMB, CAM and Spain, 1988-2014. Fuente: EVR, 1988-2014, con datos del INE
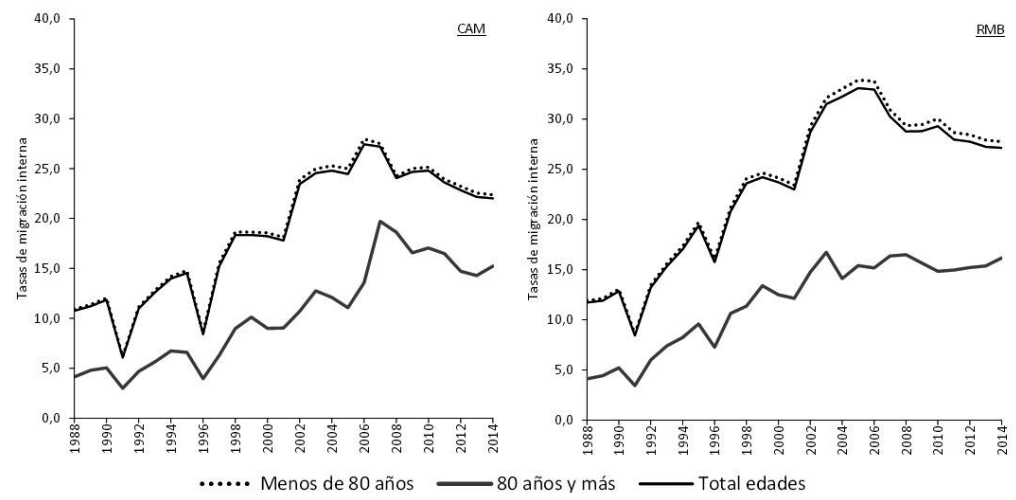

Fig. 8 - Evolución de las tasas de migración interna de los mayores y menores de 80 años y de la población total, CAM y RMB, 1988-2014.

Fig. 8 - Evolution of internal migration rates of population older and younger than 80 years, CAM and RMB, 1988-2014.

Fuente: EVR, 1988-2014, con datos del INE, Censo de población de 1991, 2001, Padrón de 1986 y Padrón continuo de población, 1996-2015 


\section{Las pautas migratorias intrametropolitanas}

La segunda pregunta de investigación plantea la existencia de pautas territoriales propias de la población mayor en comparación a otros grupos de edades o al conjunto de la población. Para responder a esta pregunta, se analizan los municipios de origen y destino de esta movilidad interna durante el bienio 2013 y 2014, últimos años con datos disponibles. Los años 2013 y 2014siguen viéndose influenciados por la crisis económica que afecta España desde 2008, aunque se observa un incremento de la movilidad de los mayores después de haber tocado fondo en 2013 en Madrid y en 2010 en Barcelona. Los efectos generales de la crisis económica sobre la movilidad metropolitana se caracterizan por un ligero descenso de la intensidad de los cambios residenciales (especialmente entre los extranjeros, pero poco importantes entre los mayores), y una atenuación de las pautas territoriales preexistentes (Pujadas et al., 2015).

Total de flujos migratorios

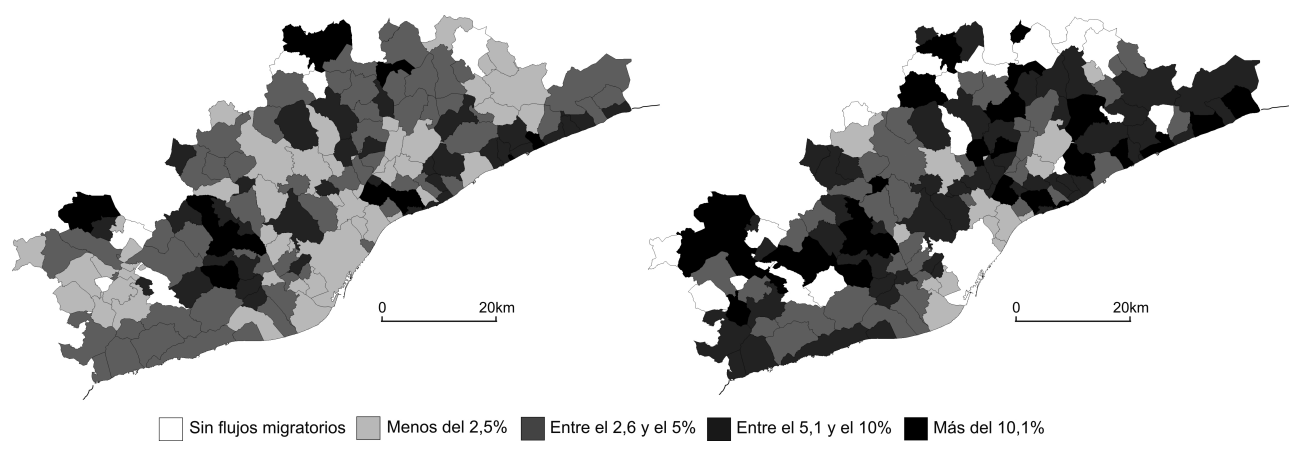

Fig. 9 - Porcentaje de mayores de 80 años en los flujos migratorios intrametropolitanos (izquierda) y en los flujos originados en el centro metropolitano (derecha), RMB, 2013-2014.

Fig. 9 - Percentage of population over 80 years in the intrametropolitan (left) and migration flows originating from the metropolitan center (right), RMB, 2013-2014.

Fuente: EVR, con datos de los años 2013 y 2014

En el caso de la RMB, en los dos años estudiados se mueven un total de 274.188 personas, 8.508 (el $3,1 \%$ ) lo hacen con 80 años o más. En primer lugar, cabe destacar el papel de la ciudad central, que de representar el $21,3 \%$ de los flujos por lugar de procedencia entre los que tienen menos de 80 años, ascienden al 30,8\% si se considera los 80 y más. Existe, por lo tanto, una sobrerrepresentación de la ciudad central en las emigraciones de los mayores. De esta forma, hasta un $4,5 \%$ de las salidas de Barcelona la producen mayores de 80 años de edad (por únicamente un 1,7\% de las entradas). De esta forma, salen 1.343 mayores y solo entran 455, con un saldo negativo de 888 personas. Este saldo representa casi una tercera parte del saldo negativo que tiene la ciudad central con su metrópolis. 
Total de flujos migratorios

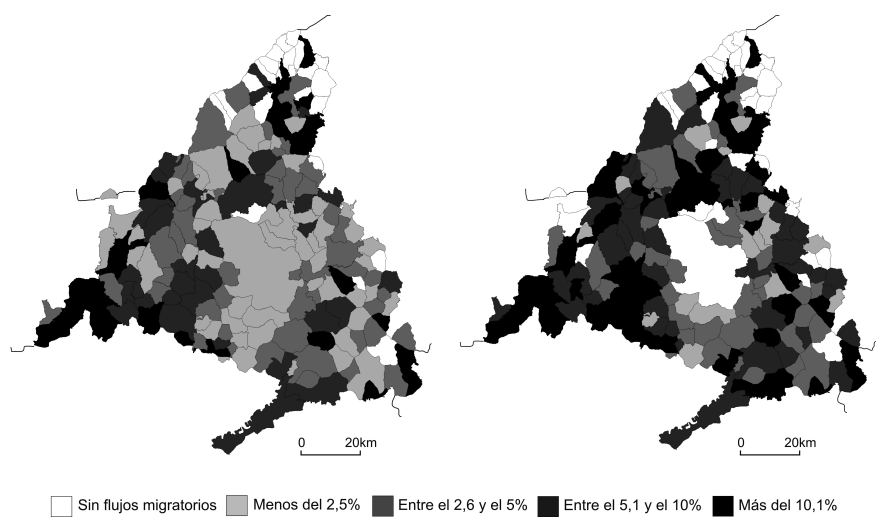

Fig. 10 - Porcentaje de mayores de 80 años en los flujos migratorios intrametropolitanos (izquierda) y en los flujos originados en el centro metropolitano (derecha), CAM, 2013-2014.

Fig. 10 - Percentage of population over 80 years in the intrametropolitan (left) and migration flows originating from the metropolitan center (right), CAM, 2013-2014.

Fuente: EVR, con datos de los años 2013 y 2014

Desde una perspectiva territorial, en la figura 9 se ha representado el peso de los mayores en los flujos recibidos por cada municipio (izquierda) y la proporción de mayores en los flujos con origen en la ciudad de Barcelona (derecha). En ellos se observa como los porcentajes menos elevados se corresponde a municipios de la primera corona metropolitana o a los de mayor tamaño situados de forma más alejada (las ciudades de Terrassa o Sabadell, por ejemplo). En cambio, en municipios de la segunda corona metropolitana de menor tamaño estos flujos se sitúan fácilmente entre el 5\% y el 10\% del total de entradas e incluso por encima, lo que nos sugiere una especialización de algunos de los municipios más alejados, de las comarcas del Maresme (Canet de Mar), o de forma muy clara entre los municipios situados en el Ordal (Vallirana, Cervelló, Corbera de Llobregat, Torrelles de Llobregat), todos ellos con porcentajes de más de un $10 \%$ de mayores entre sus llegadas. Si se consideran las salidas de Barcelona, más de 40 municipios superan el umbral del 10\%. La mayoría de estos municipios se caracterizan por tener un parque de viviendas unifamiliar importante, $y$ han experimentado durante los últimos años un fuerte crecimiento de su población por migraciones internas. Este especialización, por lo tanto, puede deberse a las dos hipótesis de trabajo, es decir, a la reagrupación familiar pero también a la institucionalización.

Para la Comunidad Autónoma de Madrid (fig. 10), entre 2013 y 2014 de los 285.402 movimientos internos, 9.479 los protagonizan el grupo estudiado, un 3,32\% de la movilidad registrada. Si consideramos la ciudad central, Madrid, las salidas de menores de 80 años representan el 29,6\% de toda la movilidad metropolitana, porcentaje que aumenta considerablemente entre los más mayores, ya que el 53,6\% de las salidas de estos se originan en la ciudad central, Madrid. La situación descrita para la RMB se produce aún con mayor intensidad en el caso de la CAM. De esta forma, hasta el 5,8\% de las salidas de la capital la protagonizan los mayores. En cuanto a su destino, existe también una cierta especialización en municipios del oeste y noroeste de la comunidad autónoma, con 52 municipios donde los mayores de 80 años representan más del 10\% de los flujos recibidos desde Madrid capital. En cambio, el poder de atracción de la capital es menor, ya que sólo un 13,9\% de los desplazamientos a estas edades se dirigen al centro metropolitano. 
Finalmente, y en relación con este último punto, destaca en ambas ciudades el hecho de que a pesar de observar algunos retornos desde la periferia al centro metropolitano, en estas edades éste no es un flujo migratorio preferente, al contrario de lo que algunas teorías sobre re-centralización podían sostener.

\section{EL ANÁLISIS DE LA MOVILIDAD INTERNA CON DATOS CENSALES}

Este último apartado de análisis busca responder la tercera pregunta de investigación, sobre la existencia de características sociodemográficas propias de aquellos que se han desplazado. Para ello se utilizan los datos del Censo de 2011, seleccionando a las personas que hayan realizado un cambio de municipio durante los últimos 10 años y que, entonces, tuvieran 80 años o más. Los microdatos del Censo 2011 permiten un mayor nivel de detalle que otras fuentes pero, como fuente para el estudio de las migraciones internas de los mayores, plantean algunas limitaciones muy claras: 1) por un lado, excluyen a las personas que se encuentran en residencias colectivas, lo que obliga a concentrarse en quienes residen en un hogar familiar; 2) por otro lado, el Censo de 2011 no es de carácter universal, sino que toma forma de una gran encuesta, lo que impone límites al número de cruces de variables que puede realizarse, afectando por ejemplo al análisis de este tipo de movilidad residencial entre colectivos de nacionalidad extranjera; 3) los efectos de la mortalidad hacen que el Censo subestime parte de los migrantes en edades avanzadas. Para las personas mayores de 80 años, la realización de un cambio residencial suele relacionarse con el empeoramiento de sus condiciones de salud. Es, por lo tanto, una población con tasas de mortalidad previsiblemente superiores a las de las demás poblaciones, lo que lleva a un subregistro por su fallecimiento durante el periodo intercensal, especialmente de aquellos que se han movido; y finalmente, 4) como en el resto de edades, únicamente disponemos de información de un cambio residencial, excluyendo la posibilidad de más de una migración durante los últimos diez años, situación que puede ser incuso muy importante entre ancianos que vivan simultáneamente en casa de varios hijos, o con un comportamiento residencial que difiera según la época del año (invierno-verano). Conjuntamente, estos factores provocan que la movilidad residencial observada con datos censales sea muy inferior a la observada con la EVR.

Tras aplicar el correspondiente factor de elevación a los microdatos del Censo 2011, obtenemos una población con 80 años o más de más de alrededor de medio millón de personas en las provincias de Barcelona y Madrid, el 23,6\% de los 2.272 .690 mayores de 80 años censados en España. De estas, el Censo estima que algo más de 99.400 en todo el estado, 15.259 en Madrid y 12.097 en Barcelona han variado su municipio de residencia en los últimos 10 años (cuando ya contaban con 80 años o más), lo que nos indica una muy baja movilidad. Esta población se ha analizado mediante un análisis de árbol que sirve para localizar aquellas variables que explican mejor la presencia o ausencia de variación residencial de mayores (cuyos resultados se incluyen en el anexo) y mediante un análisis descriptivo de las variables señaladas como relevantes tanto por el análisis de árbol complementado por la literatura existente sobre el tema.

\section{Variación residencial de mayores y características demográficas. Censo 2011}

Tanto la distribución porcentual, como el análisis de clasificación en árbol que se desarrolla más adelante, muestran la importancia que tienen los elementos demográficos. Cabe destacar la feminización y el tipo de hogar de llegada que tiene este tipo de migraciones. En proporción al total, las mujeres mayores de 80 años varían su municipio de residencia sobre un $40 \%$ más que los hombres. Esta mayor movilidad debe asociarse a su mayor longevidad, sobre todo en aquellos movimientos causados por el fallecimiento de uno de los cónyuges. 


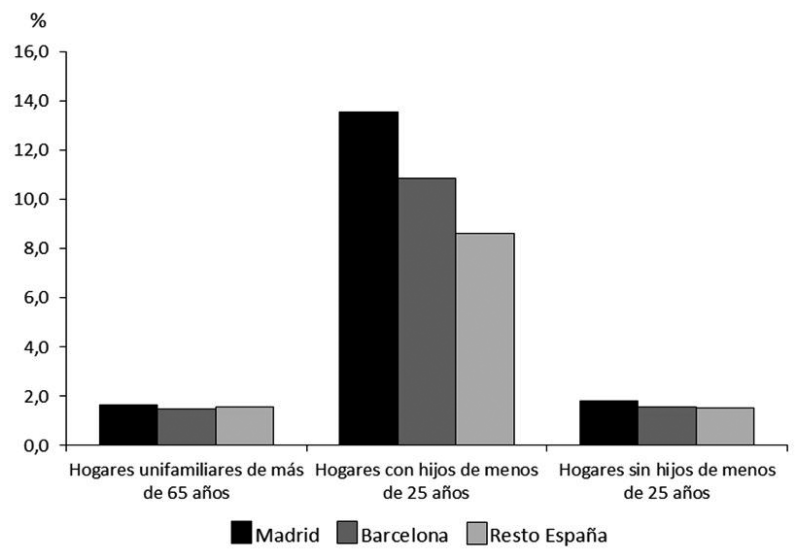

Fig. 11 - Proporción de variaciones residenciales de población mayor de 80 años en función de la estructura del hogar de destino sobre el total de variaciones residenciales de la población mayor.

Fig. 11 - Proportion of residential variations of population over 80 years, by structure of arrival household, over the total residential variations.

Fuente: Elaboración propia a partir de los Microdatos del Censo de población y viviendas 2011 (INE)

La estructura familiar del hogar de destino de la migración, en congruencia con lo que señala el estudio cualitativo de Lardíes (2010), se dirige en una mayor proporción hacia hogares familiares con hijos menores de 25 años (fig. 11). Las otras formas de hogar, todas aquellas que se pueden englobar en personas mayores solas o en hogares sin hijos menores de 25 años, no sólo presentan niveles muy inferiores en la recepción de este tipo de migración, sino que el cambio que sufren en su cruce con otras variables relevantes como provincias, tamaño de municipios, país de nacionalidad... es casi inexistente, por lo que su importancia analítica es reducida. Demográficamente, estos hogares se corresponden con los hogares de los hijos, con una corresidencia mayoritariamente con los nietos. Posiblemente, el hecho de funcionar como una unidad de cuidados hace que estos hogares estén relativamente mejor acondicionados para la llegada de un nuevo miembro y que esta llegada pueda suponer una ayuda o una carga menor al activar economías de escala.

Respecto a la estructura del hogar de partida, puede deducirse por el estado civil de la persona mayor que se desplaza, que los hogares de personas solas mayores de 65 años tienen un gran peso. Esto es, la movilidad está protagonizada básicamente por personas mayores solas. Según su estado civil, las personas solas muestran tasas más altas de movilidad que las casadas. Estas tasas de variación residencial casi se triplican al comparar la movilidad de las personas mayores viudas con las que siguen casadas (cuadro I). Este rasgo confirma algunos elementos generales del modelo que propone Rogers (1988), según el cual la movilidad está en parte determinada por las dificultades de accesibilidad de la vivienda y la soledad de la persona. Por ejemplo, en Madrid, el porcentaje de los mayores que ha variado de municipio y reside ahora en hogares con mejor accesibilidad es del 8,3\% frente a un $4,9 \%$ que no. 
Cuadro I - Porcentaje de mayores de 80 años que han variado de municipio de residencia según su estado civil y accesibilidad del edificio.

Table I - Percentage of population over 80 years who changed from municipality of residence, by marital status and accessibility of the building.

\begin{tabular}{lcccccc}
\hline & \multicolumn{3}{c}{ Accesibilidad del edificio } & \multicolumn{4}{c}{ Estado civil } \\
\hline & $\mathrm{Si}$ & No & Soltero & Casado & Viudo & Separado o divorciado \\
\hline Madrid & 8,3 & 4,9 & 6,8 & 3,0 & 8,5 & 7,7 \\
Barcelona & 4,9 & 4,6 & 6,0 & 2,5 & 6,4 & 11,3 \\
España & 5,6 & 4,0 & 5,5 & 2,5 & 6,0 & 14,7 \\
\hline
\end{tabular}

Fuente: Elaboración propia a partir de los Microdatos del Censo de población y viviendas 2011, con datos del INE

\section{Variación residencial de mayores y estructura territorial. Censo 2011}

Los diferentes porcentajes de variación residencial de personas mayores por provincias y tamaño del municipio permiten inferir el fuerte componente metropolitano de esta migración. No parece excesivamente arriesgado caracterizar estos movimientos como una recomposición de la distribución residencial activada por la incapacidad de la persona mayor, pero muy condicionada tanto por los costes de la vivienda (tanto los directos como los costes de oportunidad) en las capitales de las regiones metropolitanas como por la dispersión intermunicipal de las familias, típica de las grandes regiones metropolitanas españolas, que dificulta una atención diaria, constante y próxima a los parientes mayores. Dos elementos fundamentan esta suposición: las diferencias por tamaño del municipio de destino y las diferencias provinciales.

En primer lugar, aparece un importante efecto de capitalidad. Las grandes capitales, debido al envejecimiento de su población, densidad y coste de la vivienda (en precios de alquiler, y en oportunidades de venta) son previsiblemente expulsoras netas de población mayor a favor de los municipios menores que se encuentran en su órbita de influencia metropolitana. Así, por tamaño del municipio final, las tasas de inmigración de mayores caen por debajo del 4\% en las grandes capitales (más de 500.000 habitantes).

En segundo lugar, el porcentaje de los cambios residenciales sobre el total de población con más de 80 años es sensiblemente mayor en la provincia de Madrid (sobre el 10\%) que en la provincia de Barcelona ( $8 \%$ o menos) y, a su vez, que en el resto (sobre el $4 \%$ ). Esta gradación es paralela al porcentaje de población de la provincia residente en municipios incluidos en una dinámica de región metropolitana. Este porcentaje es casi total en la provincia de Madrid (cuyos efectos metropolitanos en ocasiones se hacen sentir en algunos municipios limítrofes de las provincias vecinas), incluye aproximadamente a la mitad de los municipios de la provincia de Barcelona y es marcadamente inferior en la mayoría de provincias del resto del Estado. 


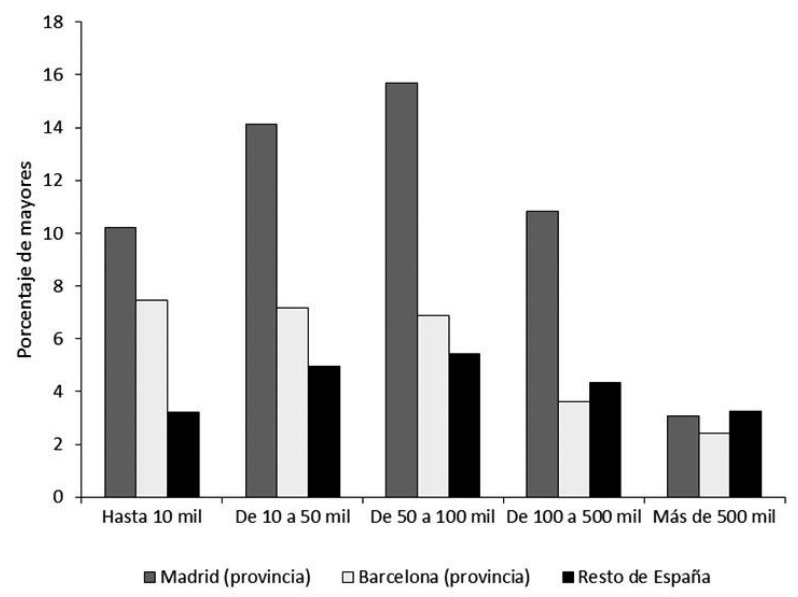

Fig. 12 - Porcentaje de mayores de 80 años que han variado de municipio de residencia según el tamaño del municipio de destino y la provincia.

Fig. 12 - Percentage of population over 80 years who changed from municipality of residence, by province and size of destination municipality.

Fuente: Elaboración propia a partir de los Microdatos del Censo de población y viviendas 2011, con datos del INE

Finalmente, el efecto de capitalidad indicado más arriba también explica por qué las tasas de inmigración de los mayores son sensiblemente inferiores en los municipios de más de 100.000 habitantes de la provincia de Barcelona (fig. 12). Esta capital, se caracteriza por tener una fuerte proporción de grandes municipios de la provincia conurbados a ella: L’Hospitalet de Llobregat, Badalona y Santa Coloma de Gramenet, por ejemplo. Esto no ocurre con otras capitales provinciales, cuya influencia metropolitana no tiene una extensión y desarrollo tan importante como la de Barcelona. Madrid, en una escala mucho mayor, sí produjo desarrollo y conurbación de sus municipios inmediatos, pero los incorporó a su término municipal durante el transcurso de su desarrollo metropolitano. Así, es en Barcelona donde existe una mayor proporción de municipios de más de 100.000 habitantes con un envejecimiento, densidad de población y precios de vivienda casi capitolinos, con sistemas migratorios propios en relación con sus municipios colindantes (Bayona \& Pujadas, 2014).

\section{Variación residencial de mayores y nacionalidad. Censo 2011}

Finalmente, la nacionalidad introduce importantes diferencias en la proporción de mayores que cambian su municipio de residencia después de los 80 años. Los mayores extranjeros presentan, sistemáticamente, porcentajes superiores de movilidad residencial (fig. 13). Un menor acceso a la vivienda en propiedad, mayor dependencia económica de las personas mayores, una mayor residencia en regiones metropolitanas, una mayor movilidad residencial, así como la posibilidad de reagrupación familiar son elementos que podrían explicar la importancia de los contrastes que produce esta variable. Además, aunque numéricamente sean poco importantes en relación a lo que sucede en otras edades, (con la única excepción de los europeos comunitarios), en su gran mayoría acaban de llegar en el último decenio a España. Esta llegada reciente no sólo es de los mayores sino del conjunto de la unidad familiar. El arrastre migratorio, en un colectivo con tasas de movilidad residencial muy importantes, explica por sí sólo su representación. Esta línea de explicación es congruente con las 
diferencias internas entre países agrupados por tipos migratorios: la población mayor de los países más desarrollados presenta tasas inferiores que el resto de nacionalidades.

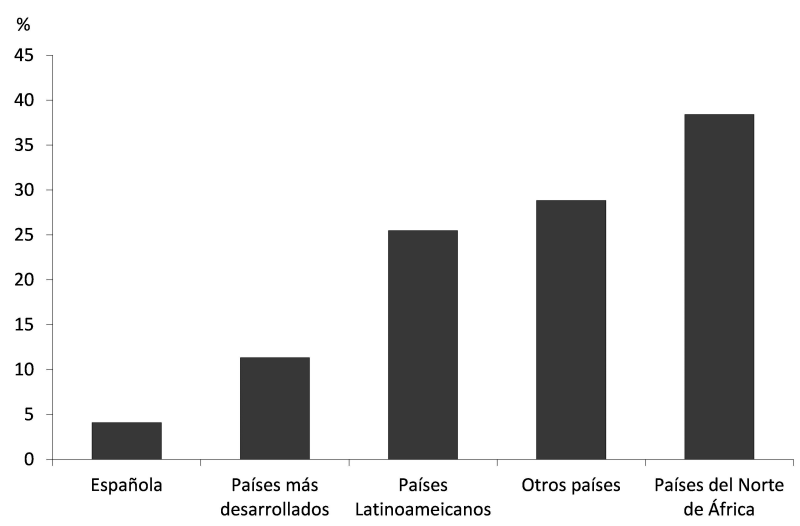

Fig. 13 - Porcentaje de mayores de 80 años que han variado de municipio de residencia según la nacionalidad.

Fig. 13 - Percentage of population over 80 years who changed from municipality of residence, by nationality.

Fuente: Elaboración propia a partir de los Microdatos del Censo de población y viviendas 2011, con datos del INE

Para concluir se incluye en el anexo (fig. 14 en anexo) la jerarquización de variables producida por la agrupación de árbol o particionamiento recursivo. Este análisis pretende explicar la presencia o ausencia de un rasgo o comportamiento en una población sobre la que se conocen también otras características relevantes. En este caso, se explica la variación residencial municipal de los mayores de 80 años. Para ello, se generan subgrupos en función de las variables independientes como la estructura del hogar, la nacionalidad, el sexo... La variable independiente que genera subgrupos con mayores diferencias porcentuales de observaciones (a partir del contraste de Chi cuadrado) es seleccionada en primer lugar. Este método se aplica recursivamente en cada nivel generando así una selección de las variables que mejor podrían explican la variación residencial de los mayores y, en ocasiones, un modelo predictivo. Se han realizado cuatro análisis: para la provincia de Madrid, para la provincia de Barcelona, para el resto de España y para el conjunto de España. Por razones de espacio, en el anexo figura únicamente este último, que recoge la información para el conjunto del país. Este análisis proporciona una selección estadísticamente significativa de las variables más determinantes, al tiempo que propone cierta jerarquización en su importancia. Así, la primera subdivisión subraya la importancia de la estructura del hogar de destino. De todos los posibles hogares familiares que pueden recibir a una persona mayor de 80 años, los más frecuentes son aquellos en los que hay hijos menores de 25 años. Después, aparece toda una serie de variables importantes a destacar: la nacionalidad, que asocia una mayor probabilidad de cambios residenciales intermunicipales a los mayores extranjeros; el tamaño del municipio de destino, que diferencia la capital y los mayores municipios metropolitanos con pocas entradas y muchas salidas del resto de municipios; el tamaño de la vivienda; el nivel de estudios; $y$, también, el estado civil (ver anexo). 


\section{CONCLUSIONES}

Los datos analizados indican como la movilidad de la población mayor es creciente, ya sea en las dos áreas metropolitanas observadas como en el conjunto del país. Por un lado, se observa la creciente movilidad residencial de la población por encima de los ochenta años, que se produce en el conjunto de España más allá de las regiones metropolitanas estudiadas. Este crecimiento es importante en números absolutos, producto del continuado envejecimiento de la población, pero también en su intensidad, como lo demuestran las tasas de migración interna. Territorialmente, las provincias con grandes aglomeraciones urbanas, pero también el norte de España, muestran altas participaciones de la movilidad intraprovincial, con valores reducidos en Andalucía y en zonas próximas a Madrid. Esta situación es aún producto de las migraciones internas españolas de mitad de siglo XX, cuyos últimos coletazos se observaban aún a inicios de los setenta.

En segundo lugar, cabe apreciar una cierta especialización territorial en el interior de las dos regiones metropolitanas estudiadas. Por un lado se reafirma el papel del centro expulsor, ya que los mayores representan un peso importante sobre las salidas de la ciudad central. Al mismo tiempo, existen municipios con altas proporciones de migrantes ancianos entre los flujos recibidos, muchos de ellos por encima del 10\% de todos los movimientos migratorios intrametropolitanos. La tipología de estos municipios nos informa de entidades municipales caracterizadas por un parque de viviendas con una alta proporción de vivienda unifamiliar y con un hábitat disperso (García et al., 2016), donde además de la recepción de migrantes metropolitanos pueden haber surgido con suma facilidad residencias de ancianos, por las características del parque inmobiliario construido.

En contraposición, el poder de atracción de las ciudades centrales es, al menos en entornos metropolitanos, insuficiente para fomentar el retorno desde las periferias de los más ancianos (lo que formaría parte de la llamada recentralización). Esta situación, en la actualidad, sería minoritaria y no justificaría el crecimiento de los flujos observados. Es más, una tercera parte de las actuales pérdidas de población de la ciudad central se estaría produciendo por el saldo migratorio negativo en edades por encima de los ochenta años de edad. Los tentáculos de la suburbanización se difuminan en el tiempo.

En último lugar, el análisis del Censo de población de 2011 ayuda a explicar las características sociodemográficas de aquellos mayores que se han desplazado. En este caso, la movilidad de los mayores hacia hogares familiares se dirige principalmente a hogares con hijos menores de 25 años (los nietos), localizados en municipios suburbanos. Además, esta movilidad es más típica en extranjeros (mayoritariamente europeos comunitarios), en mayores con nivel de estudios más elevado y en hogares que disponen de viviendas más grandes y más accesibles, lo que conjuntamente nos estaría indicando que la movilidad es más importante entre clases sociales con mayores recursos económicos. Cierta inmovilidad podría relacionarse con peores situaciones económicas y mayor vulnerabilidad (Fernández Carro \& Evandrou, 2013).

Finalmente, recordar otra vez la dificultad de registro de estos movimientos, posibilidad a veces incluso desalentada por la legislación vigente referente al proceso de herencia y a la vivienda que consta como primera residencia habitual, que desaconseja a veces el empadronamiento, y que puede funcionar con diferencias entre CCAA, clases sociales y años en función de la evolución de la legislación al respecto.

En todo caso, la reconfiguración territorial de las mayores regiones metropolitanas, con una periferización de su población, implica una mayor movilidad de arrastre de otros familiares, en este caso de mayores dependientes, que podrían estar siguiendo años más tarde el camino emprendido por sus descendientes. Esta situación es producto de la creciente complejidad metropolitana, donde el peso del centro es menor y las relaciones entre sus municipios son cada vez más intensas. Como resultado, esto comportará que aquellos mayores que se deban desplazar hacia los hogares de los hijos deban asumir, al mismo tiempo, un importante cambio en su entorno residencial, con un coste personal considerable debido a que deben romper al mismo tiempo con las relaciones sociales establecidas a lo largo de su vida. 


\section{AGRADECIMIENTOS}

El trabajo forma parte del proyecto "Desigualdad social, polarización territorial y formación de espacios vulnerables en las grandes áreas metropolitanas españolas" (CSO2015-65219-C2-1-R, MINECO/FEDER, UE)), dirigido por la Dra. Isabel Pujadas y el Dr. Fernando Gil. Los autores forman parte del Grupo de Investigación “Territori, Població i Ciutadania” (GRC_2014SGR380), reconocido por la Generalitat de Catalunya, con Isabel Pujadas como Investigadora Principal. Esta línea de trabajo dio fruto a una primera comunicación en el XIV Congreso de la Población española, en septiembre de 2014 en Sevilla (Pujadas et al., 2014). Finalmente, queremos agradecer los comentarios efectuados por los revisores, que han ayudado a mejorar el presente texto.

\section{REFERENCIAS BIBLIOGRÁFICAS}

Abellán, A. \& Rojo, F. (1997). Migraciones y movilidad residencial de las personas de edad en Madrid. Anales de Geografía de la Universidad Complutense, 17, 175-193.

Angelini, V. \& Laferrère, A. (2012). Residential Mobility of the European Elderly. CESifoEconomicStudies, 58, 544-569, doi:10.1093/cesifo/ifr017

Bayona, J. \& Pujadas, I. (2014). Movilidad residencial y redistribución de la población metropolitana: los casos de Madrid y Barcelona. EURE-Revista Latinoamericana de Estudios Urbanos y Regionales, 40 (119), 261-287. Retrived from: http://www.eure. equipu.cl/index.php/eure/article/view/358

Bayona, J. (2008). La población de nacionalidad extranjera en España: evolución y características demográficas. Entelequía: revista interdisciplinar, 8, 117-137.

Bernard, A., Bell, M. \& Charles-Edwards, E. (2014). Life-Course Transitions and the Age Profile of Internal Migration. Population and Development Review, 40 (2), 213-239.

Bonvalet, C. \& Ogg, J. (2008). The housing situation and residential strategies of older people in France. Ageing and Society, 28 (6), 753-777.

Bonvalet, C. (dir.) (2007). Vieillissement de la population et logement: les stratégies résidentielles et patrimoniales Paris, La Documentation Française (actes du séminaire Patrimoine immobilier et retraite organisé par le Ministère de l'Equipement, PUCA).

Costa-Font, J., Elvira, D. \& Mascarilla-Miró, O. (2009). Ageing in place? Exploring elderly people's housing preferences in Spain. UrbanStudies, 46, $295-$ $-316$.

Domingo, A. \& Cabré, A. (2015). La demografía del siglo XXI: evolución reciente y elementos prospectivos. In C. Torres (Ed.), España 2015. Situación social. (pp. 63-73), Madrid: Centro de Investigaciones Sociológicas (CIS).
Domingo, A. \& Houle, R. (2005). Situación laboral de la población de nacionalidad extranjera censada en España. Papers de Demografia, 266.

Fernández Carro, C. (2012). Movers or stayers? Heterogeneity of older adults' residential profiles across continental Europe. European Spatial Research and Policy, 19 (1), 17-32.

Fernández Carro, C. \& Evandrou, M. (2013). Envejecer en casa: ¿preferencia habitacional o falta de alternativas? Un análisis del contexto europeo. Comunicación al XI Congreso Español de Sociología, Madrid.

García-Coll, A., López-Villanueva, C., Pujadas, I. (2016). Movilidad residencial en tiempos de crisis. El caso de la Región Metropolitana de Barcelona. Scripta Nova, XX.

Gobillon, L. \& Laferrère, A. (2006). Les choix de logement des personnes âgées: consommation et épargne. Revue Française d'Economie, 20, 115-151.

Herbers, D., Mulder, C. \& Módenes, J. A. (2014). Moving Out of Home Ownership in Later Life: The Influence of the Family and Housing Careers. Housing Studies, DOI: 10.1080/02673037.2014.923090

Lardíes, R. (2010). Entre la movilidad residencial y la migración. Factores y razones para el cambio de residencia desde un entorno urbano entre la población mayor. Estudios Geográficos, LXXI (268), 177-201.

Méndez, R. (2008). Inmigración y mercados de trabajo urbanos: Tendencias recientes en la región metropolitana de Madrid. Scripta Nova, XII (257).

Ministerio de la Vivienda (2006). Atlas Estadístico de las Áreas urbanas en España, http://www.fomento. gob.es/mfom/lang castellano/direcciones generales/arq vivienda/suelo y politicas/atlas/

Nel.lo, O. (2004). ¿Cambio de siglo, cambio de ciclo? Las grandes ciudades españolas en el umbral del siglo XXI, Ciudad y territorio. Estudios Territoriales, 36 (141-142), 523-542. 
Parreño, J. M., Domínguez, J. \& Díaz, R. (2014). El impacto territorial de las variaciones residenciales de los jubilados españoles. In A. López-Gay et al. (Ed.), XIV Congreso Nacional de Población. Cambio demográfico y socio territorial en un contexto de crisis. Sevilla (pp. 122-136). ISBN: 978-84-697-0997-9.

Puga, D. (2000). Pautas migratorias de los mayores en España, Revista Internacional de Sociología, 27, 23-40.

Puga, D. (2004a). El comportamiento residencial de los mayores. Análisis biográfico de la movilidad en la vejez. Revista Española de Investigaciones Sociológicas, 105, 79-102.

Puga, D. (2004b). Estrategias residenciales de las personas de edad. Movilidad y curso de vida. Fundació "La Caixa”, Barcelona.

Pujadas, I., López-Villanueva, C. \& Bayona, J. (2016). Residential mobility in the Barcelona Metropolitan Region during the present economic crisis. PortugueseJournal of Social Sciences, 15 (1), 91-110.

Pujadas, I. \& Bayona, J. (2015). Las migraciones residenciales en las regiones metropolitanas de Barcelona y Madrid. In M. Domínguez \& C. López (Cords.), Barcelona y Madrid: Procesos urbanos y dinámicas sociales. Editorial Síntesis (pp. 43-70). ISBN: 978-84-907722-5-6.
Pujadas, I., Bayona, J. \& Rubiales, M. (2014). El incremento de las migraciones internas en las edades avanzadas: pautas territoriales y características sociodemográficas. In A. López-Gay et al. (Ed.), XIV Congreso Nacional de Población. Cambio demográfico y socio territorial en un contexto de crisis (pp. 150-163). Sevilla, ISBN: 978-84-697-0997-9

Recaño, J. (2004). Las migraciones internas de retorno en España durante la primera mitad de la década de los noventa: implicaciones demográficas y territoriales. Papers de Demografia, 243.

Ródenas, C. \& Martí, M. (2009). ¿Son fiables los datos de migraciones del Censo de 2001? Revista de Economia Aplicada, 50 (17), 97-118.

Rodríguez, V., et al. (2010). Inmigración y cuidados de mayores en los hogares de la Comunidad de Madrid. Informes Portal Mayores, 102.

Rogers, A. (1988). Age patterns of elderly migration: An international comparison, Demography, 25 (3), 355-370.

Rojo, F., Fernández-Mayoralas, G., Pozo, E. \& Rojo, F. M. (2001). Ageing in place: predictors of the residential satisfaction of elderly. Social Indicators Research, 54, 173-208.

Tatsiramos, K. (2006). Residential Mobility and Housing Adjustment of Older Households in Europe. Discusions Paper n. 2.435, The Institute for the Study of Labor (IZA) Bonn.

i Hacemos uso del término de "movilidad residencial" cuando hablamos de migraciones internas que se producen dentro de una misma región metropolitana.

ii Ambas fuentes son recogidas por el Instituto Nacional de Estadística (INE). Por un lado el Padrón Continuo de Población es el registro municipal que recoge a todos los residentes de un municipio (con independencia de su situación legal en el país), que se ha utilizado para obtener las poblaciones de referencia a escala municipal. Los flujos, en cambio, provienen del análisis de los microdatos de las Estadísticas de Variaciones Residenciales (EVR), que agrupa las altas y bajas que se producen en el padrón entre municipios españoles y también, aunque con menor fiabilidad, con el exterior.

iii Tanto en los casos de Barcelona como de Madrid no utilizamos las definiciones del Atlas Estadístico de las Áreas urbanas (Ministerio de la Vivienda, 2006), dibujadas para el resto de España en la figura 2, y sí que empleamos delimitaciones de mayor tamaño, que consideramos que son más apropiadas y que se utilizan comúnmente para delimitar ambas metrópolis.

iv A pesar de ello, algunos municipios de la Sierra Norte madrileña no seguirían dinámicas metropolitanas. Como su peso sobre la población total de la CAM es muy pequeño, se decide mantener como unidad de análisis el total de la comunidad.

v En España, el Censo de 2011 no tiene carácter exhaustivo, y se trata de una muestra de población. Los microdatos publicados hasta la fecha no disponen de los municipios menores de 20 mil habitantes, con lo cual es imposible reconstruir unidades territoriales por debajo de la delimitación provincial. En el caso de la provincia de Barcelona, la RMB supone el 91\% de la población provincial.

vi No se dispone de suficiente información sobre este colectivo (ni por edades ni territorial), por ello no se ha podido sumar esta información a los datos analizados.

vii Como ejemplo, basta comentar que en 2008 se alcanzó el máximo de representación de los extranjeros en la movilidad. Aquel año, en la RMB, el 41,3\% de todas las migraciones internas en fueron protagonizadas por extranjeros, mientras que si se considera los mayores de 80 años el peso de estos era de apenas de un $0,1 \%$. 


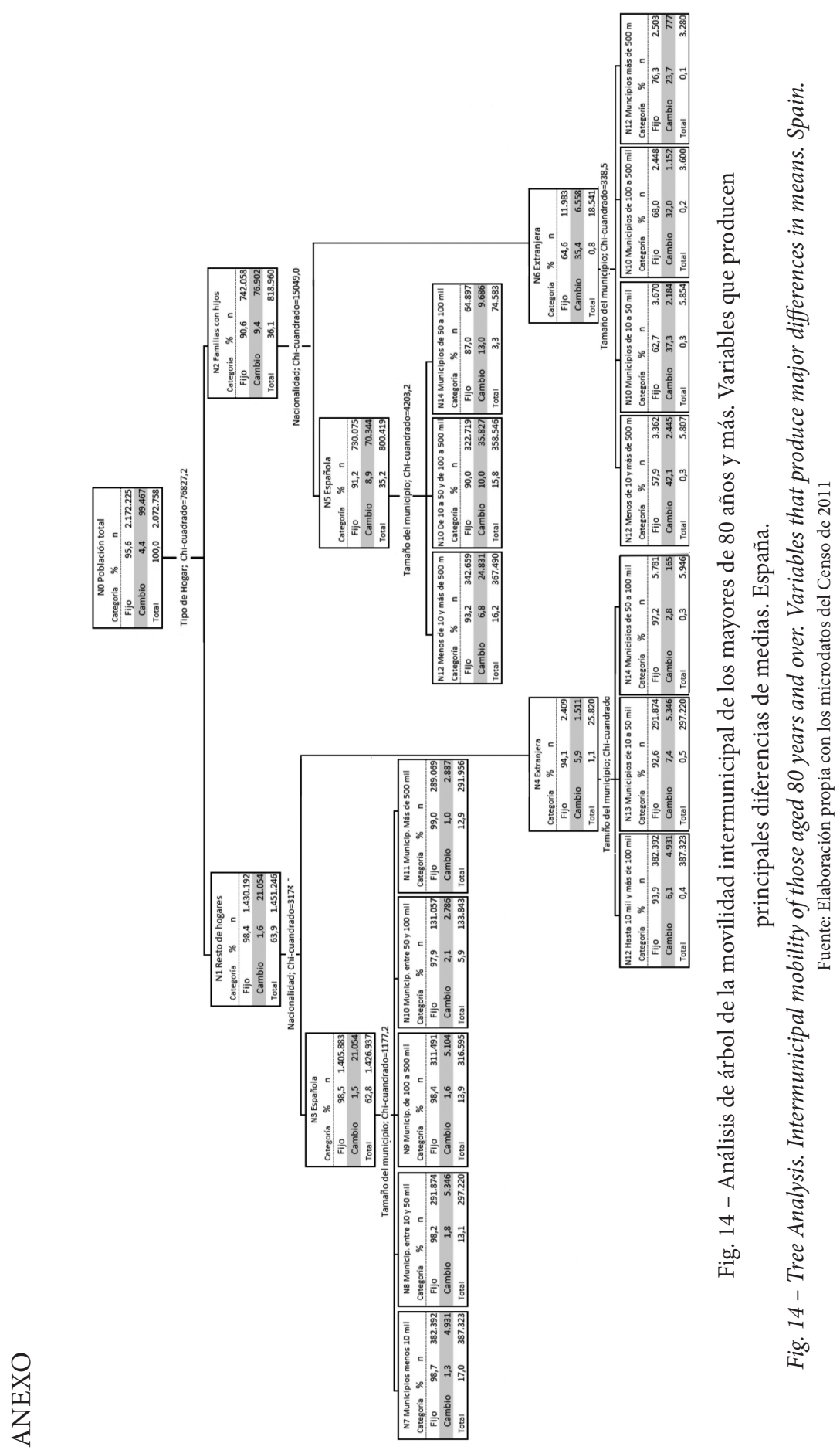

